\title{
Focal neurological deficit in acute anemia: Case reports and review of the literature*
}

\author{
Salvatore Giuffrida ${ }^{1}$, Giuseppe Carpinteri ${ }^{2}$, Daniela Modica ${ }^{1}$, Manuela Pennisi ${ }^{3}$, Alfio Catalano ${ }^{1}$, \\ Rita Bella ${ }^{1}$, Giovanni Pennisi ${ }^{1}$, Carolin Cornelius ${ }^{3}$, Giuseppe Lanza ${ }^{1 \#}$ \\ ${ }^{1}$ Department “G. F. Ingrassia”, Section of Neurosciences, University of Catania, Catania, Italy \\ ${ }^{2}$ Medicina e Chirurgia d’Accettazione d’Urgenza, "Policlinico-Vittorio Emanuele” University Hospital, Catania, Italy \\ ${ }^{3}$ Department of Chemistry, School of Medicine, University of Catania, Catania, Italy \\ Email: "giuseppelanza2003@yahoo.it \\ Received 28 February 2013; revised 29 March 2013; accepted 21 April 2013 \\ Copyright (c) 2013 Salvatore Giuffrida et al. This is an open access article distributed under the Creative Commons Attribution Li- \\ cense, which permits unrestricted use, distribution, and reproduction in any medium, provided the original work is properly cited.
}

\begin{abstract}
Introduction: Although anemia is not regarded as an usual vascular risk factor for stroke, it is one of the potential mechanism by which the brain does not receive adequate oxygenation. Moreover, the relationship between drop of hemoglobin and acute focal neurological deficits is not clear. We report two patients with cerebral infarction due to acute anemia. Case Reports: Case 1 was a 73-year-old man who complained an episode of loss of consciousness followed by right hemiparesis and dysarthria after few hours. The day after admission he presented melena caused by a duodenal ulcer bleeding. The hemoglobin dropped from $11.3 \mathrm{~g} / \mathrm{dl}$ to $5.6 \mathrm{~g} / \mathrm{dl}$ in 24 hours. Areas of acute infarctions were evident at diffusionweighted imaging (DWI) of the brain. Case 2 was a 77-year-old man with a transient episode of aphasia, right lower limb paresis and mental confusion twelve hours after an intervention of PTA and stenting of the left internal carotid artery. Hemoglobin was $11.8 \mathrm{~g} / \mathrm{dl}$ before intervention and $9 \mathrm{~g} / \mathrm{dl} 48$ hours later. DWI showed bilateral and widespread acute infarcts. Conclusion: Anemia has to be considered as a potential factor in determining or worsening cerebral infarction, especially in patients with carotid or intracranial stenosis, high cerebrovascular lesions load or insufficient collateral supply. Acute or severe anemia may negatively impact the cerebral blood flow and decrease oxygen-carrying capacity, promote rapid deterioration of ischemic penumbra. Brain DWI and treatment of the underlying etiology of acute anemia are crucial in early identification and recovery of cerebral infarctions.
\end{abstract}

*Conflict of interest: the authors do not have any conflict to disclose.

"Corresponding author.
Keywords: Low Hemoglobin Level; Focal Neurological Sign; Brain Diffusion-Weighted Imaging; Cerebral Hemodynamic; Cerebral Infarction

\section{INTRODUCTION}

Cerebral infarct results from acute interruption of oxygenated blood flow due to thrombotic or embolic occlusion or marked deprivation of nutrients for the brain, such as oxygen or glucose. Whereas hypertension, diabetes, smoking habit, old age and dyslipidemia are wellknown vascular risk factors for stroke, the role of hemoglobin value is still up for debate [1]. In 1972, the Framingham Study conducted on more than 5000 patients concluded that the pathogenetic, preventive and therapeutic implications of the interrelationship between hemoglobin level and cerebral infarction would have required further exploration [2]. The few studies investigating hematocrit value as a risk factor for stroke have produced conflicting findings [3-5] and they generally focused on the impact of a high haematocrit because of the well-known consequences on blood viscosity, oxygen-carrying capacity and possibly blood clotting characteristics $[2,6]$.

Anemia as an independent risk factor for cerebrovascular events has mainly been described in children $[7,8]$. In adults, whereas the relevance of sickle cell anemia in cerebral infarction is well depicted [9], anemia in general is not considered as an usual and definite vascular risk factor for stroke because it has been thought that the physiologic regulation in cerebral hemodynamics and oxygenation would meet the brain oxygen requirement in most circumstances, even with profound anemia [10].

However, recent studies found a significant association between anemia at the time of stroke and larger infarct territory, higher mortality over 1-year and worse 
long-term outcome [11-13]. Moreover, a recent study showed that both low and high hemoglobin levels were each associated with increased odds and worse severity of neglect, independent of stroke size [14]. Nevertheless, these aspects have only been scarcely studied in large cohorts of ischemic stroke patients and the relationship between drop of hemoglobin level and acute focal neurological deficits warrants further studies.

We report two patients with cerebral infarction due to acute anemia caused by upper gastrointestinal bleeding (Case 1) and in the context of a percutaneous transluminal angioplasty (PTA) and stenting for carotid artery stenosis (Case 2).

\section{CASE 1}

A 73-year-old man came to our attention because of a brief episode of loss of consciousness preceded by a feeling of generalized weakness. He was found confused, pale and with urinary sphincter relaxation. Transported to the Emergency Room, paleness was still evident but otherwise he appeared to be in good general health. After a few hours, he complained acute weakness at right limbs and slurred speech. All the vital signs as well as plasma glucose concentration were within normal limits. The vascular risk profile was unremarkable except for an history of hypertension under pharmacological treatment. He assumed anti-platelet drug. Neurological examination showed right hemiparesis, slight central right facial nerve paresis and dysarthria. Brain Computerized Tomography (CT) at the Emergency Room revealed a picture of subcortical cerebrovascular disease without evidence of acute ischemic or hemorrhagic stroke. The day after admission, melena was evident and the feces analysis showed active bleeding, along with a severe normocytic normochromic anemia. The hemoglobin level at baseline was 11.3 g/dl (n.v. 12 - 18) whereas, after 24 hours, it dropped at $5.6 \mathrm{~g} / \mathrm{dl}$; red blood cells were $2.87 \times 10^{6} / \mathrm{mm}^{3}$ (n.v. 4.2 - 6.1) and hematocrit value 25.2\% (n.v. 37 - 52). Other serological exams, including basic coagulation tests, routine kidney and liver function blood tests, electrolytes, iron, cardiac biomarkers and inflammatory markers, as well as urine analysis, were normal. Cardiological evaluation did not reveal specific abnormalities except for sporadic ectopic supraventricular beats. Transthoracic echocardiography demonstrated hypertensive cardiomyopathy and a slight mitral and aortic valves insufficiency. Color-coded duplex sonography of carotid and vertebral arteries revealed mild stenosis without hemodynamic change in both carotid branches. Chest $\mathrm{X}$-ray showed the heart to be enlarged and aortic arch calcifications. A brain Magnetic Resonance Imaging (MRI) was performed, including the diffusion-weighted imaging (DWI) scans. T2-weighted and Fluid Attenuated Inversion Recovery (FLAIR) showed a diffuse periven- tricular leucoaraiosis consistent with chronic cerebrovascular disease, whereas multiple bilateral subcortical areas consistent with acute infarction were evident at DWI (Figure 1). A duodenal ulcer with evidence of recent active bleeding was revealed by the endoscopic examination. Surgical intervention was excluded. The patient discontinued anti-platelet treatment and underwent to three packed red blood-cell transfusions. The anemia was corrected and the bleeding stopped, along with improvement of neurological deficit. Hemoglobin raised at $9.2 \mathrm{~g} / \mathrm{dl}$, red blood cells $3.18 \times 10^{6} / \mathrm{mm}^{3}$ and hematocrit $27.9 \%$. The patient was discharged with poor neurologic sequelae under pantoprazole, gastrointestinal cytoprotective agent and anti-hypertensive drug. No acute episodes recurred and he has remained free of any neurologic deficit after a short-term follow up.

\section{CASE 2}

A 77-year-old man was admitted because of a sudden episode of impairment of language expression, weakness at the right lower limb, mental confusion and vomiting. Twelve hours before, he was discharged for a significant stenosis of the left internal carotid artery treated with PTA and stenting. He had a history of hypertension and diabetes, both under pharmacological treatments, and he was on anti-platelet drug because of the atherosclerosis of both internal carotid arteries. Emergency brain CT did not demonstrate findings of new infarcts but evidence of chronic and diffuse subcortical cerebrovascular disease. Carotid arteries CT angiograms did not reveal restenosis or other hemodynamic abnormalities. At admission, blood pressure was $165 / 70 \mathrm{mmHg}$, heart rate $52 \mathrm{bpm}$, body temperature $36.6^{\circ} \mathrm{C}$, oxygen saturation $99 \%$ and glucose concentration by finger-stick testing $233 \mathrm{mg} / \mathrm{dl}$.

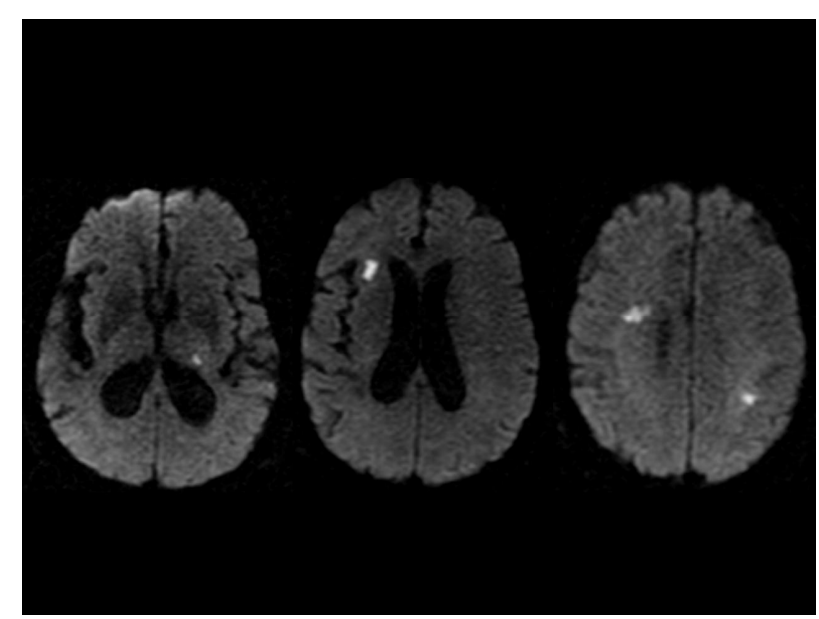

Figure 1. DWI imaging revealing small hyperintense signal abnormalities in the left thalamus (left side), in the right frontal periventricular white matter (middle) and in the centrum semiovale bilaterally (right side), consistent with recent areas of ischemic stroke. 
Repeated laboratory studies showed a moderate normocytic normochromic anemia and a trend towards an insidious decrease of hemoglobin values, from $11.8 \mathrm{~g} / \mathrm{dl}$ at the admission to $9 \mathrm{~g} / \mathrm{dl} 48$ hours later. Hematocrit value was $27.1 \%$ and red blood cells count $3.42 \times 10^{6} / \mathrm{mm}^{3}$. Transferred to our Unit, most of the neurological signs resolved spontaneously, although he appeared slightly confused. Dysarthria and right lower limb paresis lasted approximately 10 hours. Other blood tests, including white cells and platelet counts, coagulation, kidney and liver function blood tests, electrolytes, iron status, thyroid hormones and tumor markers, were unremarkable. Urine analysis resulted in a urinary tract infection, treated with a fluoroquinolone drug class. Chest X-ray was normal except for calcifications of the aortic arch. Electrocardiogram revealed normal sinus rhythm. Colorcoded carotid duplex showed diffuse atherosclerosis without hemodynamic change and stenting of left internal carotid artery. Transcranial Doppler demonstrated an accelerated blood flow at the left middle cerebral artery and a pattern of increased vascular resistance in the other examined arteries. Multiple acute infarcts were evident at brain DWI (Figure 2), in the anterior limb of left internal capsule, in the left fronto-temporo-parietal white matter, in the right part of the pons and in the cerebellum. Anemia was monitored and, at discharge, hemoglobin was stabilized at $9.4 \mathrm{~g} / \mathrm{dl}$; red blood cells count was $3.58 \times$ $10^{6} / \mathrm{mm}^{3}$ and hematocrit $28.6 \%$. Aspirin and clopidogrel were prescribed, together with anti-hypertensive and oral antidiabetic drugs. He was discharged in good general health and no further episodes of transient or persistent neurological deficit have occurred.

\section{DISCUSSION}

These reports highlight the concept that acute anemia has

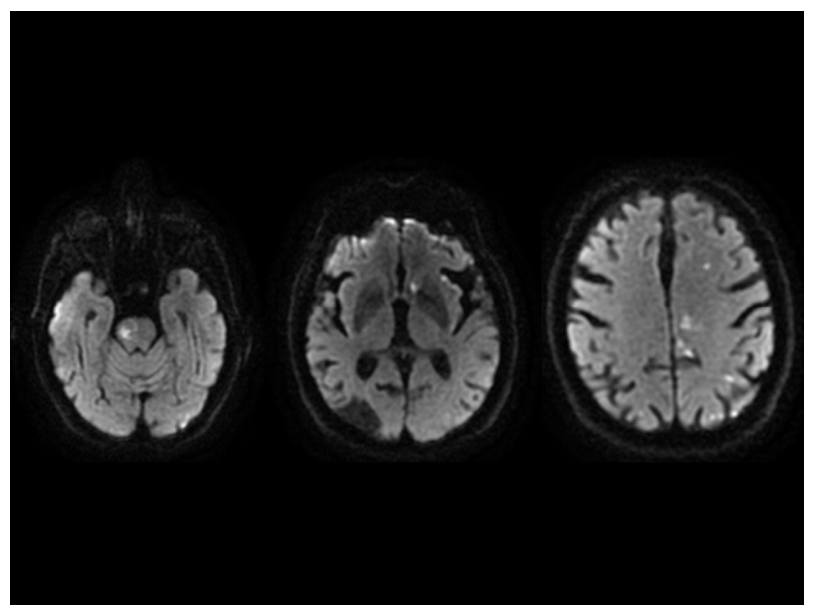

Figure 2. Acute small-size infarcts in the right part of the pons (left side), in the anterior limb of the left internal capsule (middle) and in the left fronto-temporo-parietal white matter (right side) are evident at DWI. to be considered as a potential factor in determining or worsening transient or persistent focal neurological deficit due to cerebral infarction, especially in patients with carotid or intracranial stenosis, high cerebrovascular lesions load or insufficient collateral supply. In Case 1, the close temporal relationship between the bleeding and the onset of neurological deficit suggests that bleeding and subsequent anemia may precipitate atherothrombotic cerebral infarction; in Case 2, the coexistence of anemia with a relevant hemodilution and invasive carotid artery procedures probably plays a crucial role in determining perioperative stroke.

Anemic hypoxia is the result of reduced hemoglobin content with normal oxygen tension or saturation [5]. Although other well-known factors have important consequences on neurological outcome, such as the timing of therapy, vascular territories involved, arterial pressure, blood glucose concentration and body temperature, anemia may negatively impact the cerebral circulation decreasing oxygen-carrying capacity and promoting rapid deterioration of ischemic penumbra. Moreover, as previously suggested, stroke severity may be related to hemoglobin loss course, speed and amount [15]. In addition, anemia may induce a hyperkinetic circulatory state and upregulate the endothelial adhesion molecule genes, which may lead to thrombus generation [16]. Furthermore, blood flow augmentation and turbulence may result in the migration of a thrombus that had already existed in the diseased vessel, thus producing artery-toartery embolism [16].

Along with drop of hemoglobin level, the decrease in hematocrit value might lead to a reduction in oxygen delivery relative to metabolic demand and result in high incidence of subcortical infarct. The subcortical borderzone areas were probably the most vulnerable area to ischemic injury during reduction of cerebral perfusion pressure [17]. When hemoglobin is reduced below a critical level, oxygen uptake cannot compensate adequately for increased oxygen extraction demands. Thus, the oxygen content decreased markedly in the ischemic tissue of the subcortical borderzone areas. [7] A mathematical modeling study in rabbit's brain [18] found that penumbral extraction reserve was nearly exhausted at a hemoglobin concentration of approximately $10 \mathrm{~g} / \mathrm{dl}$ and oxygen uptake in the ischemic penumbra decreased progressively when hemoglobin concentration further decreases. Therefore, a hemoglobin concentration of 10 $\mathrm{g} / \mathrm{dl}$ might represent the rational transfusion "trigger" for the acutely anemic stroke patients, although a suitable randomized clinical study has to be performed before validation of the proposed model.

Severe anemia has been also associated with transient ischemic attacks, suggesting that low haemoglobin level may accelerate the occurrence of a reversible regional 
cerebral anemic hypoxia, which is probably the consequence of a transient reduced blood oxygen-transporting capacity $[5,19]$.

The true incidence of stroke in acute anemia is probably underestimated. DWI scans for the detection of restricted diffusion areas consistent with acute infarction is recommended in patients with anemia and neurological deficits in order to early identification of cerebral ischemic lesions.

With regard to treatment, urgent blood transfusion and rapid correction of the underlying cause of anemia are the most important mandatory strategies since these patients could usually get considerable improvement after transfusion. In fact, neurological deficit often appeared in the course of acute anemia, soon after hemoglobin dropped below a critical level, and largely resolved when hemoglobin level raised [5]. No other hemodynamic factors, such as arterial hypotension or cardiac arrhythmia, that might have reduced flow below threshold levels, were present in our patients.

In conclusion, monitoring the hemoglobin and hematocrit levels is important to identify individuals who might be at higher risk for cerebral infarct and for worse functional consequences after stroke. Early recognition, rapid transfusion and treatment of the underlying etiology of acute anemia are crucial to recovery and prognosis of stroke patients. More patients' collection and awareness are required to better understand the impact and the pathophysiological relevance of acute anemia in stroke patients.

\section{REFERENCES}

[1] Tegos, T.J., Kalodiki, E., Daskalopoulou, S.S. and Nicolaides, A.N. (2000) Stroke: Epidemiology, clinical picture, and risk factors-Part I of III. Angiology, 51, 793-808. doi:10.1177/000331970005101001

[2] Kannel, W.B., Gordon, T., Wolf, P.A. and McNamara, P. (1972) Hemoglobin and the risk of cerebral infarction: The Framingham study. Stroke, 3, 409-420. doi:10.1161/01.STR.3.4.409

[3] Herold, S., Brozovic, M., Gibbs, J., Lammertsma, A.A., Leenders, K.L., Carr, D., Fleming, J.S. and Jones, T. (1986) Measurement of regional cerebral blood flow, blood volume and oxygen metabolism in patients with sickle cell disease using positron emission tomography. Stroke, 17, 692-698. doi:10.1161/01.STR.17.4.692

[4] Akins, P.T., Glenn, S., Nemeth, P.M. and Derdeyn, C.P. (1996) Carotid artery thrombus associated with severe iron-deficiency anemia and thrombocytosis. Stroke, 27, 1002-1005. doi:10.1161/01.STR.27.5.1002

[5] Shahar, A. and Sadeh, M. (1991) Severe anemia associated with transient neurological deficits. Stroke, 22, 12011202. doi:10.1161/01.STR.22.9.1201

[6] LaRue, L., Alter, M., Lai, S.M., Friday, G., Sobel, E.,
Levitt, L., McCoy, R. and Isack, T. (1987) Acute stroke, hematocrit, and blood pressure. Stroke, 18, 565-569. doi:10.1161/01.STR.18.3.565

[7] Young, R.S., Rannels, D.E., Hilmo, A., Gerson, J.M. and Goodrich, D. (1983) Severe anemia in childhood presenting as transient ischemic attacks. Stroke, 14, 622-623. doi:10.1161/01.STR.14.4.622

[8] Ganesan, V., Prengler, M., McShane, M.A., Wade, A.M. and Kirkham, F.J. (2003) Investigation of risk factors in children with arterial ischemic stroke. Annals of Neurology, 53, 167-173. doi:10.1002/ana.10423

[9] Gebreyohanns, M. and Adams, R.J. (2004) Sickle cell disease: Primary stroke prevention. CNS Spectrums, 9, 445-449.

[10] Hsiao, K.Y., Hsiao, C.T., Lin, L.J., Shiao, C.J. and Chen, I.C. (2008) Severe anemia associated with transient ischemic attacks involving vertebrobasilar circulation. American Journal of Emergency Medicine, 26, 382.e3382.e4.

[11] Nybo, M., Kristensen, S.R., Mickley, H. and Jensen, J.K. (2007) The influence of anaemia on stroke prognosis and its relation to $\mathrm{N}$-terminal pro-brain natriuretic peptide. European Journal of Neurology, 14, 477-482. doi:10.1111/j.1468-1331.2006.01591.x

[12] Huang, W.Y., Chen, I.C., Meng, L., Weng, W.C. and Peng, T.I. (2009) The influence of anemia on clinical presentation and outcome of patients with first-ever atherosclerosis-related ischemic stroke. Journal of Clinical Neuroscience, 16, 645-649. doi:10.1016/j.jocn.2008.08.014

[13] Tanne, D., Molshatzki, N., Merzeliak, O., Tsabari, R., Toashi, M. and Schwammenthal, Y. (2010) Anemia status, hemoglobin concentration and outcome after acute stroke: A cohort study. BMC Neurology, 10, 1-7.

[14] Gottesman, R.F., Bahrainwala, Z., Wityk, R.J. and Hillis, A.E. (2010) Neglect is more common and severe at extreme hemoglobin levels in right hemispheric stroke. Stroke, 41, 1641-1645. doi:10.1161/STROKEAHA.110.585265

[15] Tsai, C.F., Yip, P.K., Chen, C.C., Yeh, S.J., Chung, S.T. and Jeng, J.S. (2010) Cerebral infarction in acute anemia. Journal of Neurology, 257, 2044-2051. doi:10.1007/s00415-010-5657-6

[16] Kim, J.S. and Kang, S.Y. (2000) Bleeding and subsequent anemia: A precipitant for cerebral infarction. European Neurology, 43, 201-208. doi:10.1159/000008176

[17] Yamauchi, H., Fukuyama, H., Nagahama, Y., Shiozaki, T., Nishizawa, S., Konishi, J., Shio, H. and Kimura, J. (1999) Brain arteriolosclerosis and hemodynamic disturbance may induce leukoaraiosis. Neurology, 53, 18331838. doi:10.1212/WNL.53.8.1833

[18] Dexter, F. and Hindman, B.J. (1997) Effect of hemoglobin concentration on brain oxygenation in focal stroke: A mathematical modeling study. British Journal of Anaesthesia, 79, 346-351. doi:10.1093/bja/79.3.346

[19] Terao, S., Sobue, G., Daimaru, O., Hibino, K., Izumi, M. and Mitsuma, T. (1994) Hemodynamic TIA associated with severe anemia-a case report. No to Shinkei, 46, 879883. 